Feltes TF, eds. Moss and Adams's Heart Disease in Infants, Children, and Adolescents. 7th ed. Philadelphia, PA: Lippincott \& Wilkins; 2008:761-92.

doi:10.1016/j.jtcvs.2009.11.004

\section{THE FUNDAMENTAL} IMPORTANCE OF BASELINE COMPARISONS IN A CLINICAL TRIAL

\section{To the Editor:}

We read with interest the study by Hecht-Dolnik and associates in the September 2009 issue of the Journal, " $\mathrm{He}$ tastarch Increases the Risk of Bleeding Complications in Patients After OffPump Coronary Bypass Surgery: A Randomized Clinical Trial." In this article, they conclude that the intraoperative administration of $1 \mathrm{~L}$ of hetastarch was associated with an increase in postoperative chest tube drainage and transfusion of blood products, which resulted in early termination of the study.

Although we acknowledge the importance of the clinical question the authors have addressed, we have major concerns regarding the equality of the intervention and the control groups at baseline and the method in which this is reported. Although the authors claim that there were no statistically significant differences in baseline characteristics between groups, Table 1 indicates the baseline international normalized ratio (INR) in the hetastarch group was 1.47 (2.12) whereas in the albumin group baseline INR was 1.07 (0.10), values presented as mean (standard deviation). Although the mean value between these 2 groups may not be statistically different, it is misleading to claim baseline equivalence between the groups. The upper limit of INR is typically quoted at 1.3 , indicating the mean INR in the hetastarch group is abnormal and in the albumin group mean INR is normal. Furthermore, the large standard deviation in baseline INR in the hetastarch group can only be explained by the presence of one or more outliers with markedly abnormal baseline value. In contrast, the standard deviation for INR of the albumin group is small (0.10), indicating a typically normal distribution. We would suggest that this method of reporting baseline INR is potentially misleading. Presenting the proportion of patients in each group with abnormal INR at baseline would be substantially more informative to judge baseline equivalence of the 2 groups.

This difference in baseline INR and, by inference, bleeding risk is difficult to explain in a trial whose group assignment was by random allocation. However, in a study whose primary outcome was transfusion of blood products, understanding a difference of this nature is vitally important to interpretation of the results, particularly where transfusion practice was neither blinded nor protocol guided, as in this study.

We believe this highlights the importance of using the appropriate method of data presentation to describe baseline characteristics in a clinical trial as well as the potential pitfall of relying on $P$ values greater than .05 to indicate baseline equivalence of groups rather than clinical interpretation for the potential influence of an observed difference on the outcome of interest.

David McIlroy, MD, MClinEpi, FANZCA Ervant Nishanian, $M D, P h D$ Department of Anesthesiology Columbia University College of Physicians and Surgeons New York, NY

doi:10.1016/j.jtcvs.2009.10.058

\section{Reply to the Editor:}

We extend our thanks to Drs McIlroy and Nishanian for their careful reading of our article. Their concerns would be well placed if the average baseline international normalized ratio (INR) in the hetastarch group were 1.47. Their comment led us to review the project source data. That review revealed several transcription errors in which the decimal place in the INR was misplaced. The average baseline INR after correction of those transcription errors is 1.06 (standard deviation $=0.11$ ). This correction thus reverses the direction of the difference between the hetastarch and albumin groups in the average INR from that presented in the initial article. The statistical significance of the difference in average INR between the albumin and hetastarch groups becomes even weaker, remaining not statistically significant (Student $t=0.77 ; P=.44$, not significant).

We then investigated how many cases in each group had a baseline INR above the 1.30 threshold, as Drs McIlroy and Nishanian suggested. That threshold is crossed by 2 members $(2.6 \%)$ of the albumin group and 3 members $(3.8 \%)$ of the hetastarch group. The difference in the proportion in each group with an INR above the 1.3 threshold is not statistically significant (Fisher exact test $=1.0$, not significant).

This corrected finding supports treating the intervention and control groups in this study as equivalent. We apologize for any misunderstandings caused by this inaccuracy in the initial reported statistics summarizing and comparing baseline characteristics.

Marketa Hecht-Dolnik, $M D^{a}$
Howard Barkan, DrPH
Ananse Taharka, $M D^{a}$
John Loftus, $M D^{a}$
${ }^{a}$ Kaiser Permanente Medical Center
Oakland, Calif
${ }^{b}$ University of California
Berkeley, Calif

doi:10.1016/j.jtcvs.2009.11.031

\section{TECHNICAL OPTIONS FOR THE TREATMENT OF ANOMALOUS ORIGINS OF RIGHT OR LEFT CORONARY ARTERIES ASSOCIATED WITH AORTOPULMONARY WINDOWS}

\section{To the Editor:}

Aortopulmonary window (APW) and anomalous right coronary artery (RCA) originating from the pulmonary trunk is a rare congenital anomaly. In 


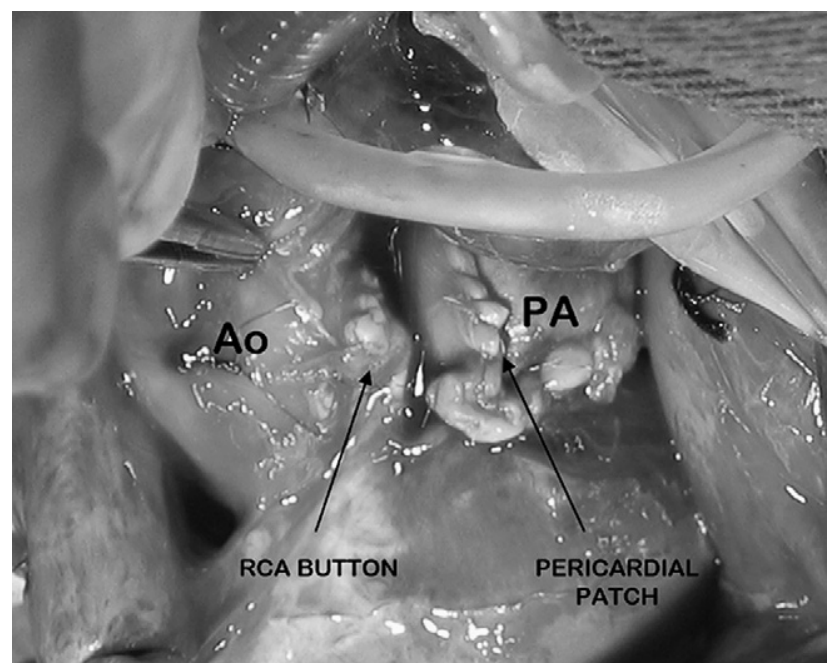

FIGURE 1. Translocated right coronary artery to the aortic side of the aortopulmonary window; pericardial patch is at the pulmonary side.

the September 2009 issue of this Jour$n a l$, Léobon and associates ${ }^{1}$ reported their clinical trial of 2 patients and options for the surgical treatment of the pathologic condition. Our group has also reported a successful surgical treatment for an infant with the same combination but with a novel technique. ${ }^{2}$

Our patient was a 4-month-old boy who had congestive heart failure and a continuous murmur on the left sternal border. Echocardiography and angiography showed APW and RCA originating from the pulmonary trunk. During the operation, after standart conduct of cardiopulmonary bypass, we made an incision in the APW toward the origin of RCA at the pulmonary end of the connection and prepared the proximal part of the RCA as a button ready for reimplantation. After ensuring that the origin of the left coronary artery was correct, we mobilized the proximal part of the RCA to avoid kinking. We then implanted the prepared RCA button to the aortic side of the transected APW with extreme care given to avoid kinking. To finish the procedure, we closed the defect at the pulmonary artery side with a pericardial patch (Figure 1). The postoperative course of the patient was uneventful with discharge on the sixth postoperative day.

The principal approach for definitive repair consists of transferring the RCA from the pulmonary artery to the aorta and closing the defect by a patch or a direct suture. ${ }^{1}$ However, in all these previosuly used methods there is always a need for an aortotomy to close the APW. Naturally, a second opening must be created for RCA implantation. Using the RCA button for closure of the defect at the aortic side may be a feasable option in patients with anatomic proximity of the RCA and APW. The most important point about this method is proximal mobilization of the RCA to avoid any kinking. We believe our succesful case suggests a different strategy for repair of this rare anomaly.

\section{Hakan Aydin} Ali Kutsal

The Department of Cardiovascular Surgery

Dr. Sami Ulus Children's Hospital Ankara, Turkey

\section{References}

1. Léobon B, Le Bret E, Roussin R, Kortas C, Ly M, Sigal-Cinqualbre A, et al. Technical options for the treatment of anomalous origins of right or left coronary arteries associated with aortopulmonary windows. J Thorac Cardiovasc Surg. 2009;138: 777-8.

2. Aydin H, Ozisik K, Surer S, Bolat A, Koc M, Kutsal A. Translocation of anomalous right coronary artery to aortic side of the aortopulmonary window: a different approach for a rare combination. J Card Surg. 2009;24:567-9.

doi:10.1016/j.jtcvs.2009.11.032

\section{Reply to the Editor:}

In their letter, Aydin and Kutsal report a new case of a right coronary artery originating from the pulmonary trunk associated with an aortopulmonary window in a 4-month old boy. They have corrected this anomaly with a modified technique of right coronary artery transfer, using the right coronary button to close the aortic wall defect. The pulmonary artery defect was closed with an autologous pericardial patch.

Owing to the anomalous site of implantation of the right coronary artery, transfer was preferable to rerouting, as suggested in our article. ${ }^{1}$ The use of the coronary artery button to close the aortic wall defect is seducing because of the quality of this autologous arterial tissue, allowing a complete repair without prosthetic material.

However, the implantation of the right coronary artery, exactly in the place where the aorta faces the pulmonary artery, may create an interaortopulmonary route of the initial part of this vessel. This could result in coronary compression in these very young patients, who may have postoperative pulmonary hypertension. Thus, my colleagues and I prefer to implant the right coronary artery where it seems, after mobilization, the most suitable on the anterior wall the aorta and to close the aortopulmonary window in a conventional manner.

Bertrand Léobon, $M D, P h D$

Department of Cardiovascular

Surgery $B$

University Hospital of Rangueil

Toulouse, France

\section{Reference}

1. Léobon B, Le Bret E, Roussin R, Kortas C, Ly M, Sigal-Cinqualbre A, et al. Technical options for the treatment of anomalous origins of right or left coronary arteries associated with aortopulmonary windows. J Thorac Cardiovasc Surg. 2009;138: 777-8.

doi:10.1016/j.jtcvs.2009.11.033 